Lecture Notes in Mechanical Engineering

Américo Azevedo Editor

Advances in

Sustainable

and Competitive

Manufacturing Systems

23rd International Conference on

Flexible Automation and Intelligent

Manufacturing 


\title{
Real-Time Tracking System for a Moored Oil Tanker: A Kalman Filter Approach
}

\author{
Paulo Malheiros, Paulo Rosa-Santos, José Gonçalves, Paulo Costa, \\ António Paulo Moreira, Fernando Veloso-Gomes \\ and Francisco Taveira-Pinto
}

\begin{abstract}
This paper presents a tracking system developed to study the behavior of an oil tanker moored at the Berth "A" of the Leixões Oil Terminal, Porto, Portugal. A brief description of the local environmental conditions and the existing operational conditions at that oil terminal are presented. Due to extreme outdoor working conditions a Kalman filter was implemented to ensure the robustness and reliability of the obtained measurements. Tests were performed in laboratory on a physical model of a moored oil tanker at a scale 1/100. The results were compared with a commercial motion capture system installed in laboratory. The presented measurement system was developed as part of the DOLPHIN project that aims to study the behavior of moored ships in harbors.
\end{abstract}

\section{Introduction}

Downtime at ocean facing ports is closely related with excessive moored ship motions caused by wave action. Reducing the amplitude of the moored ships' motions is therefore crucial to increase the on and off-loading operations efficiency, to minimize port operational costs, as well as to reduce security and environmental risks, especially when dealing with dangerous cargoes.

P. Malheiros $(\varangle) \cdot$ P. Costa $\cdot$ A. P. Moreira

INESC TEC (Formerly INESC Porto) and Faculty of Engineering,

University of Porto, Porto, Portugal

e-mail: paulo.malheiros@fe.up.pt

P. Rosa-Santos · F. Veloso-Gomes · F. Taveira-Pinto

Hydraulics and Water Resources Institute, Faculty of Engineering,

University of Porto, Porto, Portugal

J. Gonçalves

INESC TEC (Formerly INESC Porto), Polytechnic Institute of Bragança,

Bragança, Portugal 
The study of the behavior of moored ships in harbors can be justified in the initial design stage of a new port or terminal to assess the effects of some interventions on a particular berth, and also to try to improve operation and security conditions at existing berths. Usually there are three tools to deal with this issue: experience with previous and related projects, numerical modeling, and physical modeling [1]. Despite its importance for port engineering research, prototype measurements of ship motion amplitude and mooring lines and fender forces are usually scarce. Sometimes available data is not very accurate or is limited, in order to provide qualitative information. Prototype measurements are not affected by scale or laboratory effects and test conditions are realistic, although limited.

This paper presents a developed tracking system based on a physical model and stereoscopic vision data fusion, merging the data resorting to a Kalman Filter approach. The oil tanker maintains a set of hypotheses with regard to its position and the position of different objects around them. The input for updating these beliefs comes from poses belief and various sensors. An optimal estimation can be applied in order to update its beliefs as accurately as possible. After one action the pose belief is updated based on data collected up to that point in time, by a process called filtering. Kalman filtering is a standard approach for reducing the error in a least squares sense, using measurements from different sources [2-5]. The filter has two steps that are common to all localization probabilistic algorithms, which are predicted and corrected $[6,7]$. Initially it is calculated the state update based on the oil tanker model (relative measurements) and the propagation of the state covariance. Then it is included data from the developed stereoscopic vision system (absolute measurements) and the state covariance is updated.

A Butterworth linear low-pass filter was also added to the obtained data due to the knowledge that a maximum frequency of oscillation exists in the ships movements. The ship absolute position at the port terminal is measured by stereoscopic computer vision system composed of two or more synchronized cameras that can capture and record ship images from different locations from a safe distance, consequently determining its position.

The presented results are objectives of the DOLPHIN project which aims to study the behavior of moored ships in harbors and oil terminals, shown in Fig. 1. This paper focuses on the results of a simplified model of an oil terminal and surrounding area, built with the aim of analyzing the influence of an increase of the breast line pretension on the behavior of a moored oil tanker, namely on the ship motion amplitude as well as on the forces on the mooring lines and fenders.

\section{Dolphin Project}

The DOLPHIN project aims to study the behavior of moored ships in harbors and, in particular, at Berth "A" of Leixões Oil Terminal, trying to deal with its operational problems. The Port of Leixões, located in the Northwest coast of Portugal and facing the North Atlantic, has an oil terminal composed by 3 berths, 


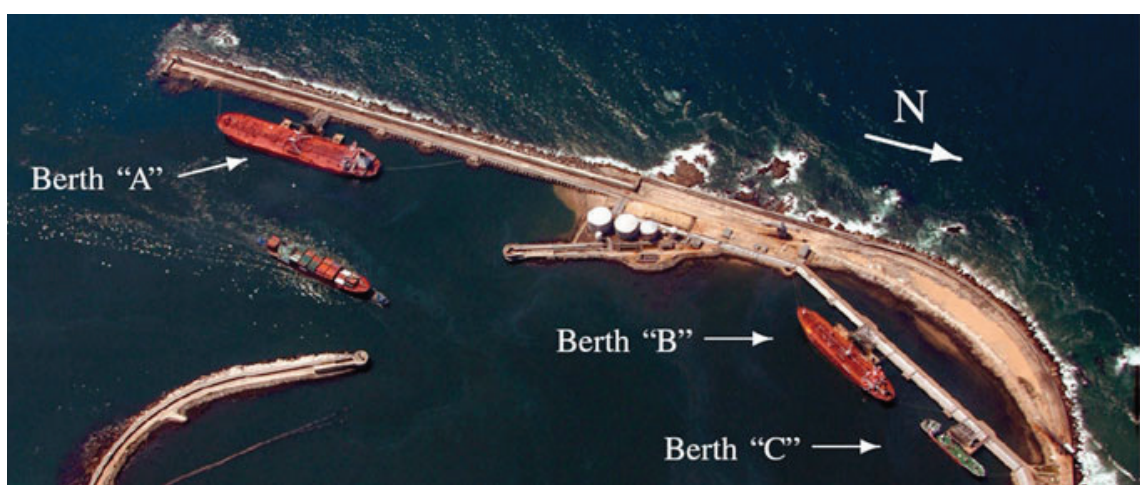

Fig. 1 Leixões Oil Terminal, Porto, Portugal

shown in Fig. 1. Berth "A", located at the harbor entrance, does not assure in average the operational and security conditions during about $20 \%$ of the time, despite the protection offered by the Leixões north breakwater [8]. In this berth the breakage of ship mooring lines can occur and moored ships sometimes have excessive movements.

The Berth "A" jetty structure consists of two breasting dolphins and a loading platform. Each breasting dolphin is equipped with a pneumatic fender and double mooring hooks. The remaining terminal mooring hooks are located on the north breakwater superstructure. Alongside this berth the bottom is about-16 mCD, which allows receiving oil tankers of up to $105,000 \mathrm{dwt}$. In the vicinity of the Port of Leixões tides are of the semidiurnal type, reaching amplitudes that range between 2 and $4 \mathrm{~m}$. The wave climate is highly energetic, the main storms come from the North Atlantic, mainly between the months of October and March. During storms significant wave heights may exceed $8 \mathrm{~m}$ and wave periods can be on the order of 16-18 s with the storm persisting for up to 5 days. Wave directions between West and Northwest prevail, also with some occurrences from Southwest.

The behaviour of an oil tanker moored at the Berth " $\mathrm{A}$ " is being studied within the scope of the R\&D project-DOLPHIN, in order to better clarify the contribution of some of the identified critical issues on Berth "A" downtime and to analyze the effectiveness of some intervention alternatives proposed in previous studies. This project includes physical model tests, numerical simulations and prototype measurement systems at Berth "A".

\section{Experimental Setup}

The physical model study was carried out at the Hydraulics Laboratory of the Hydraulics, Water Resources and Environment Division of the Faculty of Engineering of the University of Porto, on a geometric scale of $1 / 100$. The existing wave tank is $28 \mathrm{~m}$ long, $12 \mathrm{~m}$ wide, and $1.2 \mathrm{~m}$ in depth. The setup of the physical 


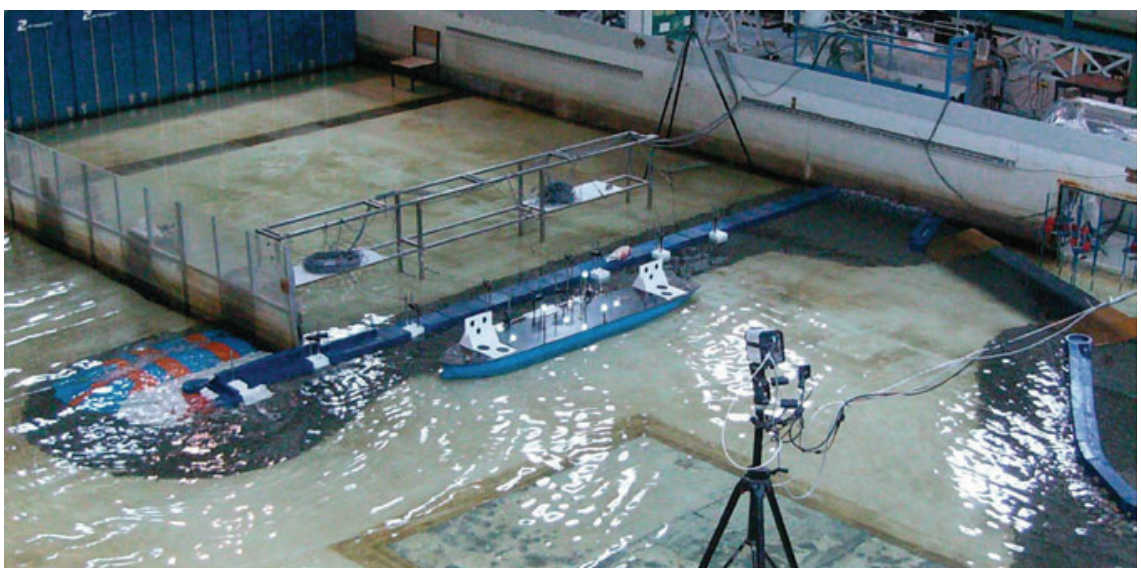

Fig. 2 The physical model in the wave tank

model in the wave tank is shown in Fig. 2. The ship selected for the study intends to represent the biggest class of oil tankers that regularly demand Berth " $\mathrm{A}$ " and corresponds to a $105,000 \mathrm{dwt}$ oil tanker, with $245 \mathrm{~m}$ overall length, $43 \mathrm{~m}$ of breath, and a maximum draft of $14.1 \mathrm{~m}$. The load-elongation curves of the ship mooring lines were simulated using a combination of precision springs, taking also into account the stiffness of the corresponding force transducer. Their non-linear behavior was linearized. This way the stiffness of each one of the mooring lines (which depends of the mooring line elongation) was replaced by the constant stiffness of an equivalent linear mooring line having the same energy absorption capacity of the non-linear mooring line. Precision springs were carefully selected to furnish the appropriate elasticity for each mooring line. The non-linear behavior of the two fenders installed was reproduced in the same way. The elasticity of each mooring element was confirmed, by calibration, prior to testing. Forces on the mooring lines and fenders were measured with suitable force transducers. The moored oil tanker motions, in the 6 degrees of freedom (surge, sway, heave, roll, pitch, and yaw), were measured using a Qualisys-Motion Capture System, composed by 3 infrared cameras [9].

One array of four wave probes was installed in the water tank parallel to the water movement, to record the water surface elevations for reflection analysis. A dissipation beach was created at the end of the wave tank to reduce wave reflections.

\subsection{Stereoscopic Vision System}

The presented system was developed to provide the absolute localization of moored ships. Carrying out measurements (in prototype) during severe environmental conditions can be very difficult or even impossible. Ideally, prototype 
measurements should also include data about wave conditions (short and long period waves) at some locations, forces on the mooring lines and fenders as well as wind and current characteristics [1].

Furthermore the restrictions imposed in such a volatile environment require the use of a passive measurement system. The development of a computer vision system to determine the ship position at the Berth "A" (Fig. 1) was based on stereoscopic vision with two or more cameras. From a safe distance, two or more synchronized cameras can capture and record ship images from different locations. With multiple cameras and choosing some ship features as natural landmarks it is possible, after a calibration process, to map the landmark pixels into points in the 3D space. The next step is to recover the ship's position and orientation from the landmark positions.

A commercial stereoscopic vision solution with three cameras provided by Qualisys was already installed in the wave tank. The developed system's cameras were installed in the vicinity of the Qualisys cameras, as shown in Fig. 3, in order to work in similar conditions. The cameras were positioned approximately at $5 \mathrm{~m}$ distance in a $45^{\circ}$ view angle. The Qualisys system consists of three infrared cameras which emit infrared light. Reflective sphere markers are placed in the ship model which allows an easy detection by the cameras. The detection of the markers is done by the cameras which mean they have internal processing. All three are connected by a cable to a personal computer (PC) and at the same time they synchronize through that same connection.

The PC records the movement through the duration of the test, a final processing is done after the end of the test presenting graphically the animated positions of the markers, as shown in Fig. 3. This system can also show the results in real-time. The position of the markers is then translated to a mathematical model of the ship which allows the calculation of the translation and orientation of the ship. The acquisition rate used for the Qualisys system during the tests was of $24 \mathrm{~Hz}$. However this system can measure up to $120 \mathrm{~Hz}$.

The developed stereoscopic system consists of two synchronized cameras, as highlighted by circles in Fig. 3, looking at the target from different positions. The

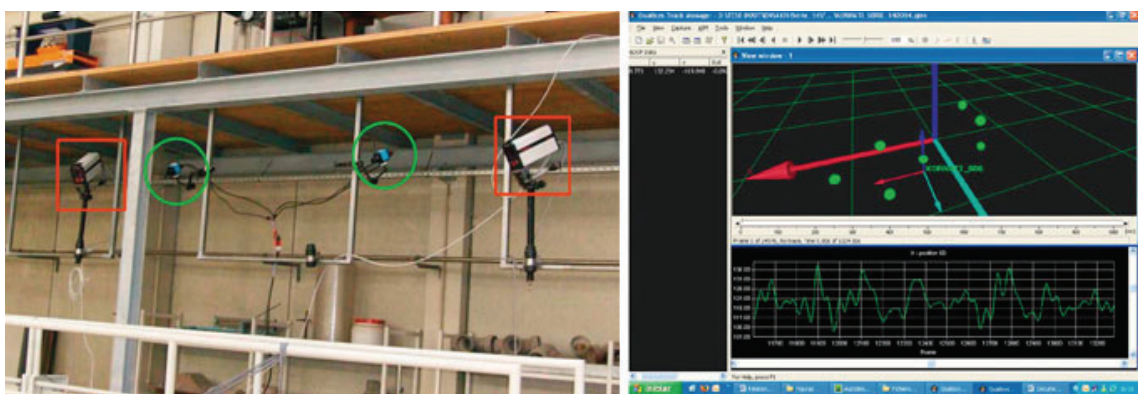

Fig. 3 Cameras installed in the Hydraulics Laboratory (on the left). Qualisys cameras shown inside squares (only two pictured), presented system cameras shown inside circles. Qualisys software markers reconstruction (on the right) 
problem consists in determining the structure of the scene from the two views, and for that two algorithms were used, the Normalized Eight-Point Algorithm [10] for the initial estimation followed by minimizing a function for fine fitting using the Levenberg-Marquardt Algorithm. The eight-point algorithm is the simplest method of computing the fundamental matrix, involving no more than the construction and (least-squares) solution of a set of linear equations [11]. If a point $X$ in space is $x$ in one image and its match $x^{\prime}$ in the second image then the fundamental matrix $F$ satisfies the condition $x^{\prime} F x=0$ (the $\mathrm{X}$ and $x$ vectors are expressed in homogeneous coordinates).

The calibration process consists of determining the cameras geometry by computing the matrix $F$. With the focal length of both views known the intrinsic parameters can be written using a $3 \times 3$ matrix $A$ and $A^{\prime}$ and assuming that the pixel in the first image is $x=A[I 0] \mathrm{X}$ where [I0] is a $3 \times 4$ matrix composed by a $3 \times 3$ identity matrix and a column of zeros. An estimate of the rotation matrix $R$ and the translation matrix $T$ can be determined as $x=A^{\prime}$ [ $[R T] \mathrm{X}$. A minimization function is then applied to achieve a metric reconstruction.

1. Solution Structure: Two FireWire CCD Bayer industrial cameras were used in this application, and an interface board with an 8-bit microcontroller was used to control the acquisition rate. Using the measurements by the Qualisys cameras a $10 \mathrm{~Hz}$ acquisition rate was found acceptable for our tests. Both visual systems work in real-time so it was ensured that the laptop had enough time to process all data in $100 \mathrm{~ms}$. Using a standard industrial camera allows to benefit from lower costs, when compared with the use of purpose built cameras.

The presented solution was developed in Object Pascal using Lazarus which is open source and runs in many operating systems. A virtual graphical representation of the scene was implemented using the GLScene components which are very useful for a fast validation of the results. The main set of components used in this application was the 5 dpo Component Library [12] developed by the 5 dpo Team for robotic applications. This library contains components for image acquisition (Firewire and USB Cameras), video display, and serial communication and is also open source.

2. The Calibration Process: The calibration of the cameras system consisted in recording a set of point matches in each image. Then with several matches (eight or more) the eight-point algorithm can determine the structure of the two views. A specific marker was designed to place on the model as shown in Fig. 4. This has circles which are easier to detect in a perspective view, and they are positioned in different planes to determine with better accuracy the various motion directions. Since these tests took several weeks this calibration process was done several times, normally more than 100 matches were used in the calibration process although the eight-point reconstruction algorithm only needed eight matches [10].

3. The Marker Detection: Two markers were placed on the bow and stern of the ship respectively as shown in Fig. 4. This was the chosen configuration because 

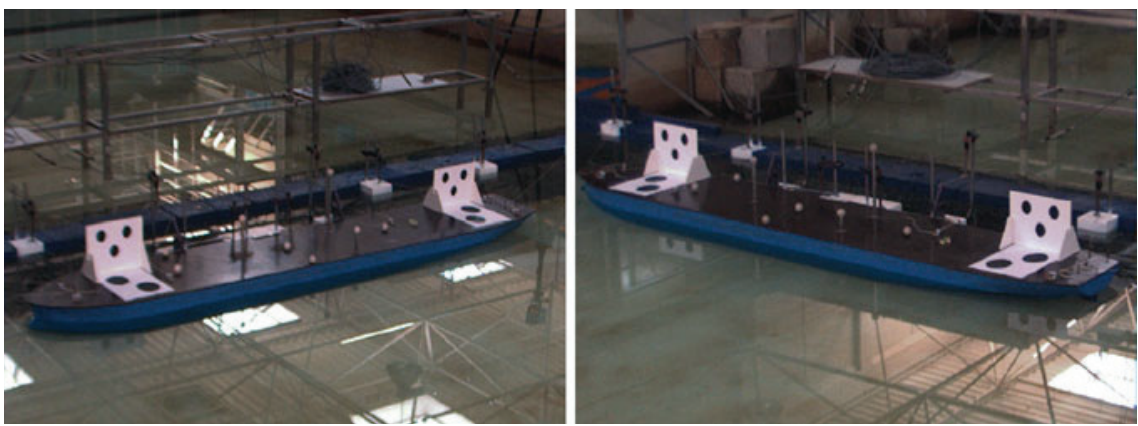

Fig. 4 Stereo images from the developed system of the physical model with markers

the remaining available space in the model was very reduced and these markers could not interfere with the markers used by the Qualisys system. These were A4 pages with a dotted pattern printed on. The markers design was chosen to achieve a precise and robust measurement, the detection of the marker consisted in two steps. The first step was to find all white rectangles in the scene and the second step was to find five black circles inside each white area. The circle centers give a very stable result. Once the markers were found each circle was classified, resulting in a match in both images.

\section{Absolute and Relative Measurements}

Having both markers 3D positions detected the final task was to determine the global position of the moored ship, in its six degrees of freedom. These are referred to its center of mass, which is approximately amidships and $100 \mathrm{~mm}$ below main deck level.

Determining the equation of the plane

$$
a x+b y+c z+d=0
$$

that is the best fit of both markers gives us the "Deck" and the vector $(a ; b ; c)$ is the normal to that plane. Going to the middle of the deck and moving in the normal direction $100 \mathrm{~mm}$ to the interior of the ship we can obtain its center of mass. This point gives us the three degrees of translation. Using the vector that unites both markers in combination with the previously obtained normal vector we can compute all three degrees of rotation.

The presented models were used to execute the first step of the Kalman filter algorithm (the state update). Different models were applied for rotation and for translation movements, as shown in the next subsections. 


\subsection{Translation Model}

The moored oil tanker when performing a translation presents a physical behavior close to a mass-spring system. Using a classical linear system the model of an object with one-dimensional movement and attached to a wall with a spring is

$$
m a=-k_{s t} x
$$

where $m$ is the mass of the object, $a$ is its acceleration, $k_{s t}$ is the spring constant and $x$ is the object position. The state equation would then become

$$
\left[\begin{array}{c}
\dot{x}(t) \\
\dot{v}(t)
\end{array}\right]=\left[\begin{array}{cc}
0 & 1 \\
-\frac{k_{s t}}{m} & 0
\end{array}\right]\left[\begin{array}{l}
x(t) \\
v(t)
\end{array}\right]
$$

where the acceleration is $a(t)=\dot{v}(t)$ and the velocity is $v(t)=\dot{x}(t)$. Difference equation in discrete form of Eq. 2 is found to be

$$
x[k]=\frac{2}{1+\frac{k_{s t}}{m} \Delta t^{2}} x[k-1]-\frac{1}{1+\frac{k_{s t}}{m} \Delta t^{2}} x[k-2] .
$$

The least-squares solution of Eq. 4 in the form $x[k]=a x[k-1]-b x[k-2]$ should yield $a \approx 2 b$ if this system model approximation was correct. Using a sample of one movement direction it was possible to determine $a=1.89$ and $b=0.89$ which was the expected result. The Kalman Filter can be implemented using the state model matrix

$$
e^{A t}=\left[\begin{array}{cc}
\cos \left(\sqrt{\frac{k_{s t}}{m}} t\right) & \frac{\sin \left(\sqrt{\frac{k_{s t}}{m}} t\right)}{\sqrt{\frac{k_{s t}}{m}} t} \\
-\sqrt{\frac{k_{s t}}{m}} \sin \left(\sqrt{\frac{k_{s t}}{m}} t\right) & \cos \left(\sqrt{\frac{k_{s t}}{m} t}\right)
\end{array}\right] .
$$

In Eq. 5 it is clear that the use of a Kalman filter allows the compensation of the system model considering the ship mass and the tension placed in the breast lines. This is one of the main reasons for using this filter for this specific outdoor application.

\subsection{Rotation Model}

A mass-spring model was also used for filtering the rotation measurements. Since these movements consist of rotations along the axes the model should depend on the moment of inertia of the ship. Assuming its shape as a cuboid one can determine the three inertial components as

$$
J_{h}=\frac{1}{12} m\left(w^{2}+d^{2}\right), J_{w}=\frac{1}{12} m\left(h^{2}+d^{2}\right), J_{d}=\frac{1}{12} m\left(h^{2}+w^{2}\right) .
$$


where the cuboid of mass $m$ has height $h$, width $w$ and depth $d$. The corresponding model is achieved identically to the translation model, shown in the previous section. The rotation state equation is

$$
\left[\begin{array}{c}
\dot{\theta}(t) \\
\dot{\omega}(t)
\end{array}\right]=\left[\begin{array}{cc}
0 & 1 \\
-\frac{k_{s r}}{J} & 0
\end{array}\right]\left[\begin{array}{c}
\theta(t) \\
\omega(t)
\end{array}\right]
$$

where $k_{s r}$ is the rotation spring constant and $J$ the corresponding moment of inertia. The value of $k_{s r}$ depends on the distance between ships center of mass and its center of buoyancy.

\section{Real-Time Tracking System Resorting to a Kalman Filter}

The used filtering system consisted of two linear filters in series, a Butterworth filter and a Kalman filter. The first was used to remove the high frequency noise from the measurements followed by a Kalman filter for real-time tracking. Observing the behavior of the moored ship (through Qualisys software) one can determine its oscillation frequency to be lower than $2 \mathrm{~Hz}$, thus the noise presence can be reduced using a low-pass filter. A fifth order Butterworth filter was implemented with the previously specified cut-off frequency.

Physical model tests were carried out with irregular long crested waves characterized by significant wave heights (Hs) of 1.5 and $2.0 \mathrm{~m}$ (on a geometric scale of $1 / 100)$, and peak wave periods (T) ranging from 10 to $18 \mathrm{~s}$. The test program included two water levels (d), namely: high tide (corresponding to a water depth, $\mathrm{d}$, equal to $20 \mathrm{~m}$ ) and mean sea level $(\mathrm{d}=18 \mathrm{~m})$. Tests were carried out with about 600 waves with the same temporal sequence for the tests having the same peak wave period. To analyze the effect of an increase of the breast line pretension two conditions were considered: "base condition", with the initial tension in all of the ship mooring lines set between 10 and $12 \mathrm{t}$; and "extra pretension condition", corresponding to a situation where the initial tension on the breast lines was increased to a value between 25 and $27 \mathrm{t}$.

The results obtained by the developed Stereoscopic Vision System (SVS) are presented in Figs. 5, 6 alongside the results from the Qualisys-Motion Capture System (QS) to allow comparison. In order to have a more clear perception of the ship motions time series, the results obtained with each of the systems were vertically shifted with QS results in the top line and SVS in the bottom line. 

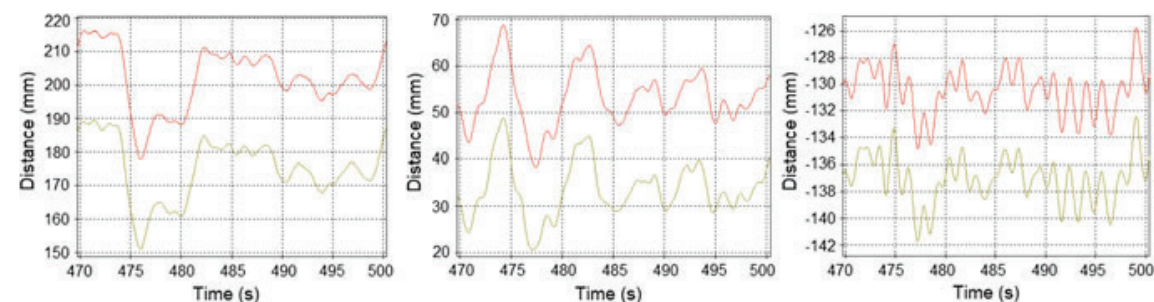

Fig. 5 Time sample of the model translation motions (surge, sway, and heave, respectively) in a test. Top line with QS results and bottom line with SVS results
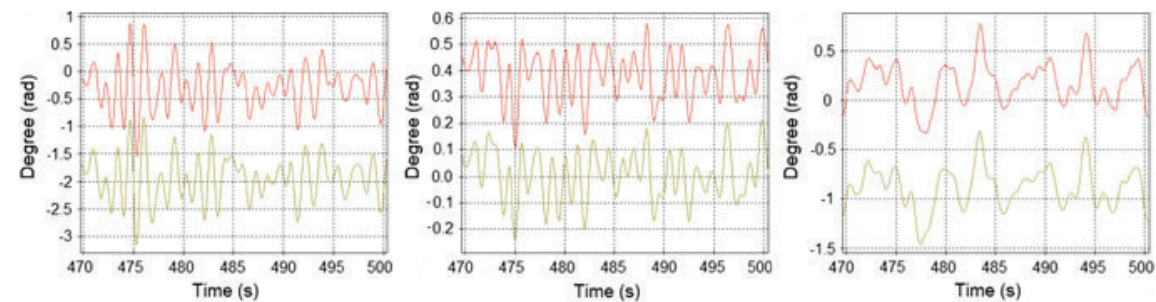

Fig. 6 Time sample of the model rotation motions (roll, pitch, and yaw, respectively) in a test. Top line with QS results and bottom line with SVS results

\subsection{Translation Motions}

The translation motions a ship can experience are the heave (up/down), the sway (side-to-side) and the surge (front/back) [13]. In Fig. 5 is represented a time sample of this ships motions. Based on Fig. 5 analysis, it can be observed that the SVS results follow correctly the values obtained with the QS and with identical magnitude.

Table 1 illustrates the maximum (Max) and significant (Signf) amplitude of the ship translation motions measured with the SVS and QS systems during the physical model test, as well as, the differences (Diff) between both systems in percentage. Significant wave height is the average wave height (trough to crest) of the one-third largest waves in a record. The SVS raw data was also added to Table 1 for analysis of the filters performance. The developed system showed consistency in all tests performed in which the sway results showed a bigger difference from the QS system. Using the filter results in better results especially in the Significant Wave, where this last one is the main characteristic in the analysis of the ship movement. 
Table 1 Translation (in meters) and rotation (in degrees) measurements

\begin{tabular}{|c|c|c|c|c|c|c|c|}
\hline \multicolumn{2}{|c|}{ Motion category } & \multicolumn{3}{|c|}{ Translation motion } & \multicolumn{3}{|c|}{ Rotation motion } \\
\hline & & Surge (m) & Sway (m) & Heave $(\mathrm{m})$ & Roll (deg) & Pitch (deg) & Yaw (deg) \\
\hline \multirow{3}{*}{$\operatorname{Max}$} & QS & 9.74 & 4.11 & 0.95 & 0.24 & 0.07 & 0.20 \\
\hline & Raw SVS & 9.73 & 4.10 & 1.00 & 0.24 & 0.07 & 0.20 \\
\hline & Diff $(\%)$ & -0.1 & 0.0 & 5.5 & 1.6 & -5.6 & 2.2 \\
\hline \multirow{3}{*}{ Signf } & QS & 2.65 & 1.92 & 0.47 & 0.11 & 0.04 & 0.09 \\
\hline & Raw SVS & 2.53 & 1.87 & 0.47 & 0.11 & 0.03 & 0.08 \\
\hline & Diff $(\%)$ & -4.7 & -2.7 & -0.9 & 0.9 & -4.7 & -2.3 \\
\hline \multirow{3}{*}{$\operatorname{Max}$} & QS & 9.74 & 4.11 & 0.95 & 0.24 & 0.07 & 0.20 \\
\hline & Filtered SVS & 9.74 & 4.11 & 1.00 & 0.24 & 0.07 & 0.20 \\
\hline & Diff $(\%)$ & 0.0 & 0.1 & 5.6 & -2.2 & -6.6 & 1.7 \\
\hline \multirow{3}{*}{ Signf } & QS & 2.65 & 1.92 & 0.47 & 0.11 & 0.04 & 0.09 \\
\hline & Filtered SVS & 2.66 & 1.91 & 0.48 & 0.11 & 0.04 & 0.08 \\
\hline & Diff $(\%)$ & 0.2 & -0.5 & 0.6 & 0.9 & -3.8 & -0.6 \\
\hline
\end{tabular}

\subsection{Rotation Motions}

The rotation motions a ship can experience are the roll (rotation about the longitudinal axis), the pitch (rotation about the transverse axis) and the yaw (rotation about the vertical axis) [13]. In Fig. 6 is represented a time sample of this ship motions. As with the translation motions these also follow correctly and with similar amplitude the results from the QS. Table 1 shows the differences (Diff) between both systems in percentage for the rotation motions where is also possible to compare the SVS raw data and the filtered tracking system. Once again the developed system showed consistency in all tests performed and the Significant Wave measurements improved with the filter approach.

\section{Conclusions}

The developed stereoscopic system was able to measure with high precision all six degrees of freedom of the model ship. All tests were developed in laboratory, resorting to a physical model of a moored oil tanker at a scale 1/100. The tests show a good agreement with the results obtained with the Qualisys system. With the cameras positioned approximately at $5 \mathrm{~m}$ distance from the target body it was possible to measure a few millimeters variations. The presented system presented a high level of scalability since it doesn't rely on personalized exterior markers since it will use the ship features to track its movement. Also the Qualisys system uses an exterior light source while the developed system works under normal ambient light. The openness of the system was one of the main concerns during the development of this system, using standard and easily accessible equipment and also with little software restrictions. 
The presented system has as future work the goal of being applied to track an oil tanker moored at the Berth "A" of the Leixões Oil Terminal, Porto, Portugal. Due to the extreme outdoor working conditions the reliability of the absolute measurements provided by the stereoscopic vision system will, inevitably, decrease. In order to minimize tracking errors, that might emerge while working outside the laboratory, a Kalman filter was implemented to ensure the robustness and reliability of the obtained measurements. It is also important, as future work, to quantify the how outdoor conditions affect the absolute measurements confidence in the measurements in order to obtain an optimal sensor fusion.

Acknowledgments The work presented in this paper, being part of the Project PRODUTECH PTI ( $n^{\circ}$ 13851) - New Processes and Innovative Technologies for the Production Technologies Industry, has been partly funded by the Incentive System for Technology Research and Development in Companies (SI I \& DT), under the Competitive Factors Thematic Operational Programme, of the Portuguese National Strategic Reference Framework, and EU's European Regional Development Fund.

The authors also thanks the FCT (Fundação para a Ciência e Tecnologia) for supporting this work trough the project PTDC/EME-CRO/114595/2009-High-Level programming for industrial robotic cells: capturing human body motion.

\section{References}

1. Taveira Pinto F et al (2008) Analysis of the behavior of moored tankers, Proceedings of the 27th international conference on offshore mechanics and arctic engineering-OMAE2008 (ASME). Estoril, Portugal, pp 15-20

2. Thrun S, Burgard W, Fox D (2005) Probabilistic robotics, MIT Press, Cambridge

3. Choset H, Lynch K, Hutchinson S, Kantor G, Burgard W, Kavrak L, Thrun S (2005) Principles of robot motion: theory, algorithms, and implementations, MIT Press, Cambridge

4. Ribeiro M (2004) Kalman and extended Kalman filters: concept, derivation and properties, technical report, IST

5. Welch G, Bishop G (2001) An introduction to the Kalman filter, Technical Report, University of North Carolina, US

6. Bravo F et al (2006) Particle-filter approach and motion strategy for cooperative localization, ICINCO

7. Rekleitis I (2003) A particle filter tutorial for mobile robot localization, International conference on robotics and automation

8. IHRH-FEUP/IST (2005) Operational conditions on the oil terminal at Leix̃oes HarbourPorto-Portugal, Technical Report, vol 6(in Portuguese)

9. Qualisys http://www.qualisys.com

10. Hartley RI (1997) In defense of the eight-point algorithm, in pattern analysis and machine intelligence, IEEE Transactions

11. Hartley RI, Zisserman A (2004) Multiple view geometry in computer vision, Cambridge University Press, Cambridge

12. 5dpo Component Library http://wiki.lazarus.freepascal.org/5dpo

13. Triantafyllou MS, Bodson M, Athans M (1983) Real time estimation of ship motions using Kalman filtering techniques, IEEE J Ocean Eng, OE-8(1):9-20 